\title{
COLOMBIA: ¿QUÉ HA PASADO CON SU REFORMA DE SALUD?
}

\section{COLOMBIA: WHAT HAS HAPPENED WITH ITS HEALTH REFORM?}

\author{
Rubén Darío Gómez-Arias ${ }^{1, a}$, Emmanuel Nieto ${ }^{2, b}$
}

\author{
Facultad Nacional de Salud Pública, Universidad de Antioquia. Medellín, Colombia. \\ Facultad Nacional de Salud Pública, Universidad de Antioquia. Medellín, Colombia. \\ Médico, MSP, phD; b economista. Magíster en Epidemiología \\ Recibido: : 30-09-14 Aprobado: 03-09-14
}

\begin{abstract}
RESUMEN
La reforma de salud adoptada en Colombia en 1993 fue promovida por diferentes agencias como el modelo a seguir en materia de política sanitaria. Siguiendo los lineamientos del Consenso de Washington y del Banco Mundial, el Gobierno de Colombia, con el apoyo de élites políticas y económicas nacionales reorganizó la gestión de los servicios de salud con base en principios del mercado, desmontó el sistema estatal, incrementó las finanzas del sector, asignó la administración del sistema al sector privado, segmentó la prestación de los servicios y promovió la interacción de los actores en un esquema de competencia de baja regulación. Después de 20 años de implantación, el modelo colombiano evidencia serias fallas y es objeto de controversia. El Estado se debilitó como ente rector de la salud; los grupos privados que administran los recursos se configuraron como fuertes núcleos de poder económico y político, y aumentaron las vulneraciones al derecho a la salud. Adicionalmente, la corrupción y el sobrecosto de los servicios han puesto en crisis la sostenibilidad del sistema, y la red estatal se encuentra en peligro de cierre. Pese a su desprestigio en el ámbito interno, varios agentes dentro y fuera del país propenden por mantener el modelo con base en reformas coyunturales.
\end{abstract}

Palabras clave: Reforma de la atención de salud, Colombia; Modernización del sector público; Financiación de la atención de la salud (fuente: DeCS BIREME).

\begin{abstract}
The health reform adopted in Colombia in 1993 was promoted by different agencies as the model to follow in matters of health policy. Following the guidelines of the Washington Consensus and the World Bank, the Government of Colombia, with the support of national political and economic elites, reorganized the management of health services based on market principles, dismantled the state system, increased finances of the sector, assigned the management of the system to the private sector, segmented the provision of services, and promoted interaction of actors in a competitive scheme of low regulation. After 20 years of implementation, the Colombian model shows serious flaws and is an object of controversy. The Government has weakened as the governing entity for health; private groups that manage the resources were established as strong centers of economic and political power; and violations of the right to health increased. Additionally, corruption and service cost overruns have put a strain on the sustainability of the system, and the state network is in danger of closing. Despite its loss of prestige at the internal level, various actors within and outside the country tend to keep the model based on contextual reforms.
\end{abstract}

Key words: Health care reform, Colombia; Modernization of the public sector; Healthcare financing (source: MeSH, NLM).

\section{INTRODUCCIÓN}

La política sanitaria colombiana suele presentarse en algunos ámbitos como el resultado de un proceso eminentemente técnico, derivado de investigaciones serias y confiables que evidencian la ineficiencia de su sistema estatal previo, y las ventajas de los modelos de mercado competitivo no regulado ${ }^{(1,2)}$. Esta reforma, sin embargo, podría comprenderse mejor como la expresión de un proceso predominantemente económico y político, donde confluyen los intereses y presiones de varios actores, influenciados a su vez por un contexto global que se ha impuesto a sus planteamientos ${ }^{(3,4)}$.

A mediados de los 80 y en seguimiento de lineamientos internacionales inspirados en el Consenso de Washington y el Banco Mundial, Colombia asumió como política la modernización del Estado y la apertura económica. Este proceso se dio en dos etapas. En primer lugar se aplicaron reformas de primera generación que propendían por: el fraccionamiento del Estado central (descentralización) y el debilitamiento de sus funciones

Citar como: Gómez-Arias RD, Nieto E. Colombia: ¿qué ha pasado con su reforma de salud? Rev Peru Med Exp Salud Publica. 2014;31(4):733-9. 
tanto reguladoras como operativas; el fortalecimiento de las finanzas públicas; la liberalización de capitales, y la preparación del Estado para subcontratar sus responsabilidades con agentes privados. La segunda fase estuvo constituida por la privatización progresiva de las funciones y recursos públicos, y la adopción del New Public Management que proponía una gestión estatal basada en los principios de la empresa privada (1). La reforma sanitaria colombiana de los 90 es una reforma de segunda generación subordinada a los cambios en la política económica; su contenido fue implantado mediante las leyes 10 de 1990, que desmontó el sistema estatal de salud, y 100 de 1993 que lo sustituyó por un sistema integral de seguridad social.

Antes de 1975 las demandas sanitarias de la población colombiana eran atendidas por un conjunto desarticulado y heterogéneo de instituciones de salud, entre las que predominaban las entidades privadas y filantrópicas financiadas mediante donaciones, juegos de azar y actividades de beneficencia. Este modelo fue incapaz de sostenerse económicamente ante una demanda de servicios cada vez mayor y fue sustituido por el Sistema Nacional de Salud (SNS) ${ }^{(5)}$ que reflejaba la concepción sistémica de los Estados europeos del bienestar. EI SNS operó entre 1975 y 1990, y concretó por primera vez en el país una estructura institucional integrada alrededor de un modelo médico asistencial de tipo estatal; dicho sistema articuló técnicamente las agencias que prestaban servicios de atención médica y ambiental en una red única de instituciones hospitalarias con diferentes niveles de complejidad, cuya construcción y operación eran financiadas y administradas por el Estado bajo un modelo de gestión vertical y centralizada, en cabeza del Ministerio de Salud. Tal centralismo mantuvo dentro de ciertos estándares la gestión de los servicios en algunas zonas especialmente deprimidas, pero interfirió con el desarrollo de las iniciativas locales de los municipios y regiones más adelantados. Los servicios se ofrecían gratuitamente a la población y se financiaban con recursos estatales que provenían, básicamente, de los impuestos directos, de venta de licores y loterías, y de rentas propias de las instituciones. A lo largo de su historia, el SNS siempre estuvo en crisis debido no solamente a la insuficiencia de recursos financieros, sino también a una gestión deficiente ${ }^{(2,6)}$.

\section{LAS REFORMAS}

Desde mediados de los 80, Colombia adoptó las políticas de modernización del Estado promovidas por las transnacionales y por los organismos financieros internacionales, (7) con el apoyo de élites locales, las cuales pretendían generar en los países de la región un espacio donde los inversionistas tuvieran acceso a los recursos y a los mercados con la máxima rentabilidad y el menor riesgo. En relación con la salud, sus promotores destacaban que las fallas del SNS para responder a las necesidades de la población, se debían a su centralismo e ineficiencia más que al modelo económico del país o a la falta de recursos financieros, y que era necesario implantar reformas estructurales ${ }^{(1)}$. Dichas reformas se enfocaron en cinco elementos complementarios: a) el incremento de recursos mediante la creación de cobros al usuario y de seguros obligatorios; b) la expansión del sector privado a áreas como la administración y prestación de servicios públicos que previamente se consideraban jurisdicción exclusiva del Estado; c) la separación del financiamiento y la provisión de servicios de salud; d) la introducción de análisis de costoefectividad como criterio para establecer prioridades de políticas y asignar recursos, y e) la retracción del Estado frente a sus responsabilidades con la salud de la población ${ }^{(1,8)}$.

En cumplimiento de estos lineamientos se promulgó la Ley 10 de 1990, que desmontó el sistema estatal de salud, fortaleció financieramente al sector y preparó a los hospitales para acceder a los recursos mediante venta de servicios. Un año más tarde, la reforma constitucional de 1991 legitimó la prestación de servicios públicos por parte del sector privado abriendo las posibilidades al mercado de la salud. Por su parte, la Ley 60 de 1993 fortaleció aun más los recursos financieros para el sector. El contenido nuclear de la reforma sanitaria se concretó en la Ley 100 de 1993, que creó el Sistema General de Seguridad Social SGSS, instaurando un sistema de atención sanitaria basado en dos principios estratégicos complementarios: la competencia regulada que ordena a los agentes estatales y privados competir por los recursos del sistema mediante la facturación de servicios, y el pluralismo estructurado ${ }^{(9)}$ que reorganiza los servicios y las relaciones entre los actores con criterios de mercado. En seguimiento de estos principios, la Ley ordenó que todo colombiano debe adquirir una póliza de seguros que cubra los costos de la atención en salud del afiliado cotizante y de su familia (beneficiarios); subordinó la atención sanitaria a pólizas obligatorias de aseguramiento que son pagadas directamente por el trabajador y su empleador mediante cotizaciones (régimen contributivo); asignó al Estado la responsabilidad de pagar las pólizas de la población más pobre (régimen subsidiado), y delegó la oferta de servicios a Instituciones Prestadoras de Servicios de Salud (IPS), de naturaleza estatal o privada, cuyas fuente de financiación dependerán de la facturación por servicios prestados. La administración de los recaudos del sistema se asignó a instituciones denominadas Entidades Promotoras de Salud (EPS); estas organizaciones son instituciones financieras 
de naturaleza privada, estatal o mixta, encargadas de recaudar anticipadamente las cotizaciones y los subsidios con base en un valor de la póliza denominado unidad de pago por capitación, cuyo monto es regulado por el Estado con base en cálculos de mercado. Las EPS tienen obligación de prestar a sus afiliados los servicios de salud requeridos, de forma directa o mediante contratación con las IPS, con base en un paquete de servicios definido denominado Plan Obligatorio de Salud POS. Las IPS, estatales y privadas, deben competir por los recursos ante las EPS, quienes les pagan los servicios prestados una vez hayan autorizado, auditado y aprobado las cuentas.

Según la Ley, los recursos no le pertenecen a la aseguradora, sino al Sistema de Seguridad Social a través de un fondo creado para tal fin que recibe el nombre de Fondo de Solidaridad y Garantías FOSYGA; la EPS está autorizada para descontar mensualmente de sus recaudos una suma que resulta de multiplicar el número de afiliados y beneficiarios por el valor de la UPC, y el monto restante lo debe remitir al fondo común FOSYGA, quien le reconoce la diferencia en caso de que los recaudos no le alcancen para cubrir las UPC de sus afiliados y beneficiarios. Con el valor de las pólizas que le corresponden por año, la aseguradora debe garantizar a sus afiliados y beneficiarios los paquetes de servicios que define la ley; esta es legalmente su responsabilidad, y si su gestión es deficiente, la aseguradora puede quebrar. La población que no tiene seguro recibe el nombre de "población vinculada" pues no existe acto administrativo que señale su afiliación, y debe ser atendida en las instituciones estatales con cargo a la autoridad municipal o departamental. Como resultado de la reforma, se medicalizó el cuidado de la salud y los servicios fueron asumiendo la figura de mercancías de alta, mediana o baja rentabilidad. Las acciones colectivas y de salud pública cuyo efecto deletéreo dificulta precisar quién debe pagar por ellas se asignaron a las autoridades territoriales quienes deben prestarlas con base en un paquete de intervenciones gratuitas denominado Plan de Atención Básica.

\section{ASPECTOS FINANCIEROS DE LA REFORMA DE SALUD EN COLOMBIA}

Las políticas de modernización del Estado inyectaron recursos frescos al sector y lo hicieron atractivo para los inversionistas. Entre 1980 y 1992, hubo un crecimiento sostenido de recursos del sector público destinados a la salud, con un incremento promedio anual de 3,3\% (7). Para 1993, más de 7,3\% del PIB de Colombia, público y privado, se destinaba a salud ${ }^{(10)}$. El situado fiscal destinado al sector se elevó del $15 \%$ en 1975 al
$23 \%$ en 1994 y al 25,5\% en $1996^{(11)}$. Desde la reforma el gasto público en salud ha venido aumentando progresivamente pasando de 1,4\% del PIB en 1988 a $3,7 \%$ en 2001 . El incremento de recursos reactivó en Colombia el negocio de los seguros (11). Las EPS pasaron de recaudar las cotizaciones de un 20,6\% de la población en 1993, al 62\% en 2004. Para 1999, 41\% de colombianos cotizaban al Régimen Contributivo y $21 \%$ estaban cubiertos por el régimen subsidiado ${ }^{(12)}$. Al ponerse en marcha la reforma, los empresarios que administraban los recursos del sector fueron asumiendo un papel hegemónico en la gestión de la política sanitaria y en poco tiempo las aseguradoras, consolidadas como uno de los sectores económicos de mayor desarrollo en el país, fortalecieron su poder financiero, traspasaron su negocio inicial y mediante mecanismos de integración vertical incursionaron en la prestación directa de los servicios de salud con lo que mejoraban sus ganancias $(13,14)$. En concepto del economista William Sciao, consultor para las reformas, el poder político de las aseguradoras es actualmente muy grande en Colombia y se basa en su poder financiero y sus vínculos con las élites políticas y económicas ${ }^{(15)}$.

Paralelamente, la política de adelgazamiento del Estado debilitó los sistemas control estatal sobre la dinámica del sector que se rige cada vez más por procesos de oferta y demanda orientados por el sector privado. El sistema de atención sanitaria experimentó un proceso rápido de fragmentación que se revela en múltiples procesos de segmentación: de los agentes según sus competencias, de los paquetes de servicios según su rentabilidad y de los usuarios según su capacidad de pago. La fragmentación orientada por el mercado derivó también en una pérdida del know how y un debilitamiento de la calidad y efectividad de los servicios, lo que llevó a su vez al deterioro de indicadores como el control de la malaria, la TB, la mortalidad materna, la fiebre amarilla, el dengue y otras enfermedades ${ }^{(3,16,17,18)}$. No se dispone de estudios amplios de calidad de los servicios, pero los reclamos de los usuarios podrían actuar como indicador; entre 1999 y junio de 2002 se presentaron en el país más de 500000 recursos judiciales de amparo al derecho a la salud (en Colombia se denominan acciones de tutela) que fueron interpuestos por los ciudadanos ante los jueces, y la cuarta parte de ellos reclamaba servicios de salud que habían sido negados por las EPS. En 2012 se registraron 454500 acciones de tutela de las cuales el $25 \%$ correspondían a reclamos de los ciudadanos por vulneraciones al derecho a la salud, esto equivale a una tutela cada cuatro minutos ${ }^{(19)}$.

La búsqueda de la eficiencia, considerada el principal criterio para asignar los recursos y evaluar las políticas, es uno de los pilares de la reforma. 
Paradójicamente, los costos de la intermediación generados por las aseguradoras son precisamente uno de los elementos que más debate ha suscitado en el país ${ }^{(20)}$. La búsqueda de eficiencia se ha convertido en un argumento de las administradoras para aumentar su rentabilidad; las EPS contratan por volumen, solicitando descuentos, subcontratando a destajo con otros intermediarios, conteniendo costos y aumentando sus utilidades, pero a costa de la evasión de sus obligaciones con el usuario, y del esfuerzo no pagado a los prestadores y trabajadores de la salud. En diferentes informes, los organismos de control han advertido que en esta cadena de intermediaciones gran parte del dinero no llega al usuario. Adicionalmente, la corrupción ha impregnado el sistema de salud considerado como uno de los negocios más dinámicos e importantes del país. En resumen, la reforma ha recibido mucho más dinero, sin evidencia de mejores resultados sobre la salud de la gente, lo que obedece a la desviación de fondos, la corrupción y la falta de control estatal ${ }^{(21,22)}$.

A diciembre de 2013, los recursos destinados a la salud ascendían en Colombia a 34 billones de pesos equivalentes a USD 16 870,3 millones (Tabla 1). Las fuentes principales correspondían a cotizaciones establecidas sobre nómina $(52,2 \%)$, seguidas de los aportes fiscales canalizados a través del Sistema General de Participaciones (SGP) y los excedentes financieros del Fosyga. Entre 2010 y 2013 estos recursos se destinaron a financiar el régimen contributivo $(44,7 \%)$; el régimen subsidiado $(33,1 \%)$; la prestación de servicios a población pobre no asegurada $(7,4 \%)$; los recobros por medicamentos No Pos y los fallos judiciales de tutela (8,0\%); y la salud pública $(4,4 \%)$.

Los datos oficiales sugieren un aumento del financiamiento del $7,68 \%$ anual entre 2010 y 2013 , basado fundamentalmente en el crecimiento de los excedentes del Fosyga y las cotizaciones provenientes del aporte del $12,5 \%$ sobre nómina; este hallazgo podría considerarse razonable en el contexto del ciclo ascendente de la economía nacional y los superávits de la cuenta de compensación del régimen contributivo. Sobre los rubros presentados inciden de manera negativa el carácter estructural del empleo precario, la informalidad laboral y los bajos salarios en el país. En este sentido, no hay que perder de vista que en el período de referencia la densidad salarial, es decir, el salario base de cotización, se ha mantenido en cerca de 1,8 del salario mínimo legal.

Los recursos del SGP, es decir las transferencias del nivel nacional a los entes territoriales que son soporte del régimen subsidiado, crecen anualmente en no más del $3,23 \%$ como consecuencia de la regla fiscal que en 2011 elevó a rango constitucional el control del déficit fiscal, y también de la reforma constitucional del 2000 que desligó dichas transferencias de los ingresos corrientes del fisco central. Desde 1994 hasta ese año las transferencias crecieron anualmente en 10\%. Entre 2001 y 2012, por el contrario, las transferencias para salud aumentaron solo en un $3 \%$ anual ${ }^{(23)}$. Pese a las limitaciones de orden estructural que pudiera estar soportando el flujo del financiamiento de la salud en Colombia, lo cierto es que su ritmo de crecimiento y la magnitud de los recursos no son para nada despreciables, especialmente si se contrastan con lo registrado en el contexto latinoamericano (tabla 2) donde se registran las estadísticas del gasto en salud en valores per cápita y como proporción del producto interno bruto en los países de la región. Como proporción del PIB, el gasto en salud aumentó sustancialmente de 1995 a 2012 en la mitad de estos países, sobre todo en Brasil, Costa Rica, Ecuador y Paraguay. En el resto se mantuvo estable; este es el caso de Colombia que presenta el nivel medio de América Latina con la particularidad de que, junto con Bolivia y Costa Rica, registra en el último año las proporciones más altas del gasto de naturaleza pública.

Tabla 1. Fuentes de recursos del Sistema General de Seguridad Social en Salud, Colombia 2010-2013 (millones de dólares americanos calculados sobre pesos, constantes de 2013)

\begin{tabular}{|c|c|c|c|c|c|c|}
\hline \multirow{2}{*}{ Fuentes } & \multicolumn{4}{|c|}{ Millones de dólares americanos } & \multirow{2}{*}{$\begin{array}{c}\text { Distribución } \\
\%\end{array}$} & \multirow{2}{*}{$\begin{array}{c}\text { Crecimiento } \\
\%\end{array}$} \\
\hline & 2010 & 2011 & 2012 & 2013 & & \\
\hline Cotizaciones & 7100,3 & 7622,1 & 8234,5 & 8662,2 & 52,2 & 6,9 \\
\hline SGP & 2891,2 & 2915,4 & 3025,9 & 3178,9 & 19,9 & 3,2 \\
\hline Excedentes Fosyga & 1605,6 & 1258,8 & 1446,8 & 1883,0 & 10,2 & 7,8 \\
\hline Resto & 1934,1 & 2450,8 & 3319,2 & 3146,1 & 17,7 & 17,6 \\
\hline Total fuentes & 13531,2 & 14247,1 & 16026,4 & 16870,3 & 100 & 7,68 \\
\hline
\end{tabular}

Tasa de cambio a Sept 28 de 2014: un dólar americano USD= 2015,11 pesos colombianos; SGP: Sistema general de participaciones; Fosyga: Fondo de Solidaridad y Garantías

Fuente: Ministerio de salud. Cifras financieras del sector salud. Boletín bimestral N. ${ }^{\circ} 1$, diciembre de 2013 
Tabla 2. Gasto en salud en valores per cápita y proporción del PIB en países de América Latina, 1995 y 2012

\begin{tabular}{|c|c|c|c|c|c|c|}
\hline \multirow{2}{*}{ Países } & \multicolumn{3}{|c|}{1995} & \multicolumn{3}{|c|}{2012} \\
\hline & $\%$ PIB & \% Público & US\$ p-c & $\%$ PIB & $\%$ Público & US\$ p-c \\
\hline Argentina & 8,3 & 59,8 & 616 & 8,5 & 69,2 & 995 \\
\hline Bolivia & 4,5 & 63,8 & 39 & 5,8 & 71,8 & 149 \\
\hline Brasil & 6,7 & 43,0 & 316 & 9,3 & 46,4 & 1.056 \\
\hline Chile & 6,5 & 38,2 & 334 & 7,2 & 48,6 & 1.103 \\
\hline Colombia & 6,8 & 55,1 & 171 & 6,8 & 75,8 & 530 \\
\hline Costa Rica & 6,5 & 76,5 & 219 & 10,1 & 74,6 & 951 \\
\hline Ecuador & 3,4 & 55,4 & 73 & 6,4 & 44,8 & 361 \\
\hline México & 5,1 & 42,1 & 177 & 6,1 & 51,8 & 618 \\
\hline Paraguay & 5,9 & 36,0 & 112 & 10,3 & 42,0 & 392 \\
\hline Perú & 4,5 & 54,4 & 100 & 5,1 & 58,9 & 337 \\
\hline Venezuela & 4,2 & 42,6 & 143 & 4,6 & 33,7 & 593 \\
\hline
\end{tabular}

p-c: per cápita

Fuente: Banco Mundial. Página oficial http://datos.bacomundial.org/indicador

La pregunta relevante para el caso colombiano es si el volumen actual de recursos en salud pudiera considerarse suficiente para garantizar el acceso a los servicios al conjunto de la población. El gasto per cápita estimado por el Banco Mundial permite un primer acercamiento a dicho interrogante. En efecto, en Colombia dicho gasto ascendió en 2012 a USD 530, equivalente a \$ 953062 pesos colombianos, los cuales superan los \$547000 que valía para ese año la Unidad de Pago por Capitación (UPC), la cual es justamente el valor reconocido por el SGSSS a las Empresas Promotoras de Salud (EPS) para garantizar el Plan Obligatorio de Salud (POS) a cada uno de sus afiliados. Colombia cuenta hoy, como nunca antes en su historia, con un monto apreciable de recursos para la salud de su población. Sin embargo, este esfuerzo no se compadece con los indicadores en salud ni con las barreras impuestas a la población para acceder a los medicamentos y servicios requeridos. Lo anterior se relaciona de forma estrecha con el destino que han padecido dichos recursos. En este campo se han documentado los altos costos de intermediación impuestos al sistema por las EPS, por encima no solo de los observados en países de América Latina sino incluso en países de la OCDE, (24) así como los costos de transacción derivados del incumplimiento de contratos de esas mismas instituciones, el cual explica en Colombia el creciente número de amparos judiciales al derecho ${ }^{(25)}$ y un enorme pasivo con las instituciones prestadoras de servicios. El tema se relaciona con el carácter que han asumido las EPS en el sistema de salud, las cuales han transformado su papel de garantes de servicios de calidad a gestoras del riesgo financiero o a meras intermediarias entre los usuarios y las IPS.

En su calidad de ordenadoras del gasto, las EPS han gozado, desde los inicios de la reforma, de un poder de mercado indiscutible a lo largo del país, especialmente en regiones periféricas y de baja densidad poblacional donde el mercado de aseguramiento tiende a asumir un carácter monopólico. Esta posición dominante ha permitido a las EPS no solo imponer condiciones contractuales a las IPS sino también alinear con sus intereses a los organismos rectores, cooptando las instancias de regulación y sometiendo al sistema a altísimos costos de administración, permitiéndose, incluso, actuaciones por fuera de la normatividad; los efectos perversos de esta práctica, que han sido objeto de investigaciones y sanciones por parte de los organismos estatales de control, no solamente han atentado contra la sostenibilidad financiera del sistema sino también contra los recursos públicos y los derechos fundamentales de los usuarios. En 2011 la Superintendencia de Salud inició investigación a 14 EPS por haber acordado una política de negación de servicios incluidos en el POS, forzando con ello acciones de tutela por parte de los usuarios. Dichos servicios terminaban siendo recobrados al Fosyga a tarifas incluso superiores a las del mercado, con lo cual dichas EPS obtenían recursos adicionales a la UPC ${ }^{(26)}$.

Ante los fallos de rectoría, vigilancia y control del sistema, buena parte de las EPS han adoptado un ejercicio sistemático de captura de rentas y actuaciones dolosas en contra de las finanzas del sistema de salud, algunas de las cuales solo en años recientes vienen siendo ventiladas por la Superintendencia de Salud, los organismos de control del Estado y los estrados judiciales. Los desvíos de dineros y los fraudes al sistema de salud por parte de las EPS vienen de tiempo atrás. Así lo estableció en 2013 la Contraloría General de la República con un fallo de responsabilidad fiscal por valor de 1,4 billones de pesos (USD 695 millones) contra la EPS más grande del sistema. En este mismo año, la Fiscalía General de la Nación estableció que 


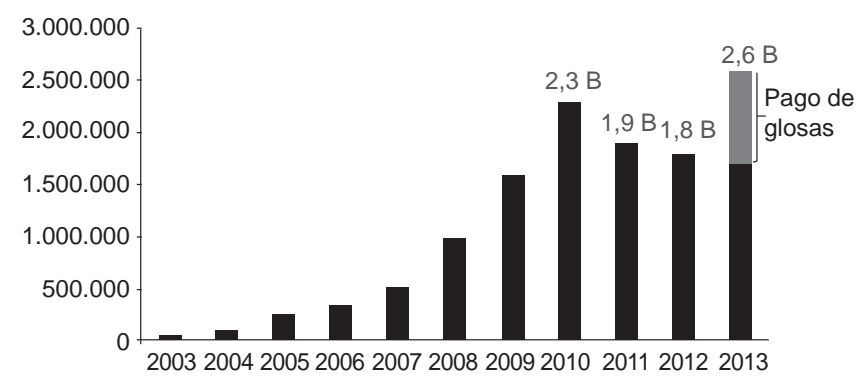

Figura 1. Pagos del Fondo de Solidaridad y Garantías- Fosyga por recobros no incluidos en el Plan Obligatorio de Salud- POS, Colombia 2003-2013 (Millones de pesos)

Fuente: Ministerio de Hacienda. Boletín de Seguridad Social. Bogotá 2014

las directivas de esta misma EPS realizaron de manera fraudulenta cerca de 10000 recobros al Fosyga; para ese entonces el organismo de fiscalización había establecido que en los últimos 10 años varias EPS habían tramitado de manera irregular no menos de 40 000 recobros al Fosyga ${ }^{(27)}$. No son pocos los dineros extraviados en la maraña de intermediarios del sistema. El monto de los recobros efectuados al Fosyga por las EPS aduciendo servicios y medicamentos no incluidos en el POS, buena parte de ellos injustificados, alcanzaron en 2010 una cifra record de 2,3 billones de pesos (USD 1141,4 millones); el escándalo nacional dio lugar a la expedición de circulares de la Comisión de Precios de Medicamentos fijando valores máximos de recobros que hicieron descender este rubro (Figura 1). Pese a que las autoridades juzgan atípicos los montos de 2013, en tanto que incorporan glosas de extemporaneidad y/o pagos por recobros de vigencias anteriores, lo cierto es que el valor de dichos recobros por parte de las EPS continúa en niveles sensiblemente altos.

Lo anterior pudiera ser indicativo de que las medidas adoptadas por las autoridades de regulación, vigilancia y control no han sido del todo eficaces, pues algo similar continúa sucediendo con los pasivos que distintos actores del sistema mantienen con la red prestadora de servicios. La Asociación Colombiana de Hospitales y Clínicas ha estimado que para diciembre de 2013, las cuentas por cobrar suman cerca de 5 billones de pesos colombianos (USD 2481,3 millones), el 54\% de los cuales constituyen cartera morosa, monto que representa una proporción superior a la cartera morosa de 2010 . El $65,8 \%$ de estas obligaciones corresponde a deudas contraídas por las EPS en ambos regímenes, subsidiado y contributivo; ${ }^{(28,29)}$ con el agravante de que buena parte de esa cartera no podrá ser cancelada por sus titulares en tanto que algunas de esas EPS están en causal de liquidación y/o intervenidas por la Superintendencia de Salud.

Las fallas del modelo se han vuelto tan evidentes y su desprestigio tan grande que el sistema es prácticamente insostenible. En los últimos años el Gobierno nacional ha recibido múltiples presiones de diferentes sectores, incluyendo la Corte Constitucional, la Contraloría, la Procuraduría y la Defensoría del Pueblo, quienes denuncian la incoherencia e inconveniencia del modelo y propenden por una reforma estructural que se ajuste a los principios del Estado de Derecho, proteja la salud de la población y defienda los intereses públicos de la voracidad de los inversionistas; sin embargo los grupos económicos son políticamente muy poderosos en Colombia y los últimos intentos de reforma han terminado en modificaciones accidentales que no tocan el control de los recursos por los intermediarios.

\section{CONCLUSIONES}

En la práctica, la reforma colombiana de 1993, inspirada por las políticas neoliberales, ha movilizado grandes sumas desde el bolsillo de los contribuyentes y de los consumidores nativos, generando un mercado alrededor de las necesidades sanitarias que es aprovechado por los inversionistas nacionales y extranjeros. Pese al incremento del gasto el modelo muestra fallas importantes de calidad, efectividad y eficiencia. El afán por apropiarse de las utilidades económicas de este mercado es, en el fondo, el factor que ha impulsado y mantenido el modelo sanitario de Colombia a pesar de sus evidentes fallos y su desprestigio.

Conflictos de interés: RDGA declara que comparte los principios del Movimiento por el Derecho a la Salud.

Fuentes de financiamiento: estrategia de sostenibilidad 20132014 de la Universidad de Antioquía. Grupo de Epidemiología.

\section{REFERENCIAS BIBLIOGRÁFICAS}

1. González A, Mogollón O. La reforma de salud y su componente político: un análisis de factibilidad. Gac Sanit. 2002; 16(1):39-47.

2. Jaramillo-Pérez I. El futuro de la salud en Colombia ley 100 de 1993, política social, mercado y descentralización. 2 da ed. Santa Fé de Bogotá; Fescol: 1995.

3. Gómez-Arias RD; Universidad de Antioquía. La mortalidad evitable como indicador de desempeño de la política sanitaria. Colombia, 1985-
2001. Medellín: Universidad de Antioquia; 2008.

4. Ugalde A, Homedes N. Las reformas neoliberales del sector de la salud: déficit gerencial y alienación del recurso humano en América 
Latina. Rev Panam Salud Publica. 2005; 17(3):202-9.

5. Colombia, Congreso de la República. Decreto Ley 056 de 1975, por el cual se define el Sistema Nacional de Salud. No 34260. Bogotá: Congreso de la República; 1975.

6. Yepes-Luján F; Colombia, Ministerio de Salud. La salud en Colombia. Estudio sectorial de salud. Bogotá: MINSA; 1990

7. World Bank. Colombia - Towards increased efficiency and equity in the health sector: an decentralization help? Washington, D.C.: World Bank; 1994.

8. Armada F, Muntaner C, Navarro V. Health and social security reforms in Latin America: the convergence of the World Health Organization, the World Bank and transnational corporations. Int J Health Serv. 2001;31(4):729-68.

9. Londoño JL, Frenk J. Structured pluralism: towards an innovative model for health sector reform in Latin America. Health Policy. 1997 Jul;41(1):1-36.

10. Harvard University School of Public Health. Report on Colombia health sector reform and proposed master implementation plan. Final report to the Ministry of Health. Boston: Harvard School of Public Health; 1998.

11. Bossert T, Chawla M, Bowser D, Beauvois J, Giedion U, Arbeláez J, et al. Applied research on decentralization of health systems in Latin America: Colombia case study. Boston: Harvard School of Public Health; 2000.

12. Colombia, Ministerio de Salud. Informe de actividades al Congreso de la República 1998-1999. Bogotá: MINSA; 1998.

13. González-Rossetti A, Bossert T. Mejorando la factibilidad política de la reforma en salud: un análisis comparativo de Chile, Colombia y México. Boston: Harvard School of Public Health; 2000.

14. González-Rossetti A, Ramírez P. Enhancing the political feasibility of health reform: the Colombia case. Boston: Harvard School of Public Health; 2000.

15. Ronderos MT. Lo mejor y más débil del sistema de salud colombiano. Revista Semana. 2009; Julio 6.

16. Arbelaez MP, Gaviria MB, Franco A, Restrepo R, Hincapié D, Blas E. Tuberculosis control and managed competition in Colombia. Int J Health Plann Manage. 2004 Oct-Dec;19 Suppl 1:S25-43.

17. Kroeger A, Ordoñez-Gonzalez J, Aviña AI. Malaria control reinvented: health sector reform and strategy development in Colombia. Trop Med Int Health. 2002 May;7(5):450-8.

18. Ross A, Zeballos J, Infante A. La calidad y la reforma del sector salud en América Latina y el Caribe. Rev Panam Salud Publica. 2000;8(1/2):93-8.

19. defensoria.gov.co [Internet]. Bogotá: Defensoría del Pueblo; 2014 [actualizado el 23 de noviembre del 2014]. Disponible en: http://www. defensoria.gov.co/

20. La tutela y los derechos a la salud y la seguridad social 2013. Bogotá. La Defensoría. www.defensoria.gov.co

21. Martínez F. La crisis de la seguridad social en salud en Colombia ¿Efectos inesperados del arreglo institucional? Bogotá: Fedesalud; 2013.

22. Contraloría General de la República. Economía Colombia. Contraloría General de la República. Sistema Nacional de Salud: inmensamente rico en su miseria. Economía Colombiana No 336. Bogotá: Contraloría General de la República; 2012.
23. Colombia, Procuraduría General de la Nación. Financiamiento del Sistema General de Seguridad Social en Salud. Seguimiento y control preventivo a las políticas públicas. Bogotá: ECOE; 2012.

24. Guerrero R, Prada S, Chernichovsky D. La doble descentralización en el sector salud: evaluación y alternativas de política pública. Cali: PROESA; 2014.

25. Organización Panamericana de la Salud. Análisis comparativo de los costos de transacción, administración o intermediación de los sistemas de salud en algunos países de América Latina y de la OCED. Washington DC: OPS; 2007.

26. Nieto E, Arango A. Costos de los servicios de salud tutelados y del proceso legal de las tutelas en Medellín, 2009. Rev. Fac. Nac. Salud Pública. 2011;29(3):223-31.

27. González LE. Catorce EPS serían multadas por hacer 'pacto' para negar servicios. El Tiempo. 24 de abril de 2011.

28. Redacción Justicia. Fiscalía comprobó irregularidades en 40 mil recobros a varias EPS. El Tiempo. 20 de febrero de 2013.

29. Grupo de investigaciones ACHC. $31^{\text {a }}$ Informe de seguimiento de cartera hospitalaria con corte a diciembre 31 de 2013. Hospitalaria. 2014 MarAbr;94:4-21.

Correspondencia: Rubén Dario GómezArias.

Dirección: Facultad Nacional de Salud Pública Universidad de Antioquia. Medellín. Colombia

Teléfono: 57(4)2196890

Correo electrónico: rubengomez33@gmail. com 